\title{
Solar-powered oxygen delivery for the treatment of children with hypoxemia: protocol for a cluster-randomized stepped- wedge controlled trial in Uganda
}

Nicholas Conradi ${ }^{1}$, Qaasim Mian', Sophie Namasopo ${ }^{2}$, Andrea L. Conroy ${ }^{3}$, Laura L. Hermann ${ }^{4}$, Charles Olaro ${ }^{5}$, Jackson Amone ${ }^{5}$, Robert O. Opoka ${ }^{6}$ and Michael T. Hawkes ${ }^{1,7,8,9,10^{*}}$ (i)

\begin{abstract}
Background: Child mortality due to pneumonia is a major global health problem and is associated with hypoxemia. Access to safe and continuous oxygen therapy can reduce mortality; however, low-income countries may lack the necessary resources for oxygen delivery. We have previously demonstrated proof-of-concept that solar-powered oxygen $\left(\mathrm{SPO}_{2}\right)$ delivery can reliably provide medical oxygen remote settings with minimal access to electricity. This study aims to demonstrate the efficacy of $\mathrm{SPO}_{2}$ in children hospitalized with acute hypoxemic respiratory illness across Uganda.

Methods: Objectives: Demonstrate efficacy of $\mathrm{SPO}_{2}$ in children hospitalized with acute hypoxemic respiratory illness. Study design: Multi-center, stepped-wedge cluster-randomized trial. Setting: Twenty health facilities across Uganda, a low-income, high-burden country for pediatric pneumonia. Site selection: Facilities with pediatric inpatient services lacking consistent $\mathrm{O}_{2}$ supply on pediatric wards. Participants: Children aged $<5$ years hospitalized with hypoxemia (saturation <92\%) warranting hospital admission based on clinical judgement. Randomization methods: Random installation order generated a priori with allocation concealment. Study procedure: Patients receive standard of care within pediatric wards with or without $\mathrm{SPO}_{2}$ system installed. Outcome measures: Primary: 48-h mortality. Secondary: safety, efficacy, $\mathrm{SPO}_{2}$ system functionality, operating costs, nursing knowledge, skills, and retention for oxygen administration. Statistical analysis of primary outcome: Linear mixed effects logistic regression model with 48-h mortality (dependent variable) as a function of $\mathrm{SPO}_{2}$ treatment (before versus after installation), while adjusting for confounding effects of calendar time (fixed effect) and site (random effect). Sample size: 2400 patients across 20 health facilities, predicted to provide $80 \%$ power to detect a $35 \%$ reduction in mortality after introduction of $\mathrm{SPO}_{2}$, based on a computer simulation of $>5000$ trials.
\end{abstract}

Discussion: Overall, our study aims to demonstrate mortality benefit of $\mathrm{SPO}_{2}$ relative to standard (unreliable) oxygen delivery. The innovative trial design (stepped-wedge, cluster-randomized) is supported by a computer simulation. Capacity building for nursing care and oxygen therapy is a non-scientific objective of the study. If successful, $\mathrm{SPO}_{2}$ could be scaled across a variety of resource-constrained remote or rural settings in sub-Saharan Africa and beyond.

Trial registration: Clinicaltrials.gov, NCT03851783. Registered on 22 February 2019.

Keywords: Solar oxygen, Pediatric pneumonia, Hypoxemia

\footnotetext{
*Correspondence: mthawkes@ualberta.ca

'Department of Pediatrics, University of Alberta, 3-588D Edmonton Clinic Health Academy, 1140587 Ave NW, Edmonton, Alberta T6G 1C9, Canada

${ }^{7}$ Department of Medical Microbiology and Immunology, University of

Alberta, Edmonton, Canada

Full list of author information is available at the end of the article
}

(c) The Author(s). 2019 Open Access This article is distributed under the terms of the Creative Commons Attribution 4.0 International License (http://creativecommons.org/licenses/by/4.0/), which permits unrestricted use, distribution, and

reproduction in any medium, provided you give appropriate credit to the original author(s) and the source, provide a link to the Creative Commons license, and indicate if changes were made. The Creative Commons Public Domain Dedication waiver (http://creativecommons.org/publicdomain/zero/1.0/) applies to the data made available in this article, unless otherwise stated. 


\section{Background}

Globally, approximately 7.7 million children per year die before the age of five years. Infectious diseases account for a large proportion of these deaths, with pneumonia being the leading cause of mortality (2.1 million deaths/ year) [1]. Most deaths occur in resource-constrained settings in Asia and Africa [2]. These countries report 2-10 times more children with pneumonia than industrialized countries [3]. In Uganda alone, child mortality is estimated to be 145,000 deaths per year [4]. Bacterial pneumonia, tuberculosis, sepsis, and severe malaria are common infectious etiologies, all of which lead to respiratory distress as a final common pathway. Oxygen $\left(\mathrm{O}_{2}\right)$ therapy is essential to support life in these patients.

Large gaps remain in the clinical management of children presenting to African hospitals with respiratory distress, including essential supportive therapies such as supplemental $\mathrm{O}_{2}$. Despite being listed on the World Health Organization's (WHO) list of essential medicines [5], $\mathrm{O}_{2}$ may not be available in hospitals and health centers in low- and middle-income countries (LMICs) because of cost and/or logistical challenges [6, 7]. Methods currently used in low-resource settings include compressed $\mathrm{O}_{2}$ cylinders and grid-powered $\mathrm{O}_{2}$ concentrators $[8,9]$. Cylinders require supply chains linking $\mathrm{O}_{2}$ production plants to hospitals which may be compromised by poor road conditions, costs of transportation, and unstable supply chain management $[8,9] \cdot \mathrm{O}_{2}$ losses from cylinders can also vary greatly due to leakage $[10,11] . \mathrm{O}_{2}$ concentrators, while shown to be more cost-effective and userfriendly than cylinders, depend upon a reliable and uninterrupted supply of electricity which is often unavailable in resource-constrained settings $[8,10]$. A previous systematic review showed that $26 \%$ of health facilities in sub-Saharan Africa reported no access to electricity while only $28 \%$ of centers reported reliable access [12].

In resource-constrained settings, $\mathrm{O}_{2}$ delivery systems can lead to measurable improvements in survival from childhood pneumonia. A multi-hospital effectiveness study in Papua New Guinea demonstrated a reduction in mortality from childhood pneumonia from $5.0 \%$ to $3.2 \%$ (35\% reduction in mortality) after implementation of an enhanced $\mathrm{O}_{2}$ delivery system [13]. We have previously described and implemented a novel strategy for $\mathrm{O}_{2}$ delivery that could be implemented in remote locations with minimal access to an electrical power supply: solar-powered oxygen $\left(\mathrm{SPO}_{2}\right)$ delivery $[14,15]$.

$\mathrm{SPO}_{2}$ is an effective solution for supplemental $\mathrm{O}_{2}$ in low-resource settings [14-16]. Our systems have been described in detail previously and implemented at two hospitals in Uganda [14-16]. In brief, photovoltaic cells installed on the roofs of hospitals collect solar energy, which is stored as electricity in a battery bank, then used to power an $\mathrm{O}_{2}$ concentrator for production of medical grade $\mathrm{O}_{2}$. We previously demonstrated the feasibility, safety, and efficacy of $\mathrm{SPO}_{2}$ through a proof-of-concept study and a randomized controlled trial (RCT), showing clinical non-inferiority compared to cylinder $\mathrm{O}_{2}$ [14-16]. We enrolled 130 children with hypoxemia admitted to two Ugandan hospitals and showed that the length of stay was not prolonged in patients randomized to $\mathrm{SPO}_{2}$, relative to children randomized to cylinder $\mathrm{O}_{2}$ [15]. Further, we did not detect statistically significant differences in mortality between $\mathrm{SPO}_{2}$ and cylinder $\mathrm{O}_{2}$. Given its efficacy, before the widespread implementation of $\mathrm{SPO}_{2}$ across Africa and Asia, an evaluation of its impact on decreasing the mortality of children admitted with acute hypoxemic respiratory illness is required.

\section{Methods and design Objectives}

This study aims to demonstrate the efficacy of $\mathrm{SPO}_{2}$ in children hospitalized with acute hypoxemic respiratory illness in Uganda. The study is a multicenter prospective evaluation of $\mathrm{SPO}_{2}$, using a stepped-wedge clusterrandomized design.

Our primary aim is to compare the 48-h mortality among children aged $<5$ years admitted with hypoxemia before and after the implementation of $\mathrm{SPO}_{2}$ delivery at 20 hospitals in Uganda, adjusting for confounding effects of calendar time and site. As secondary aims, we will compare safety and efficacy outcomes among participants, monitor $\mathrm{SPO}_{2}$ system functionality and operational costs, and build capacity among nurses to deliver $\mathrm{O}_{2}$. The working hypothesis is that $\mathrm{SPO}_{2}$ can decrease the mortality of children admitted with hypoxemia Additional files 1 and 2 .

\section{Study design}

The study will be a stepped-wedge cluster RCT. This trial will not be blinded. Clusters (health facilities) will be randomly allocated to the timing of implementation of the $\mathrm{SPO}_{2}$ system. The installation of $\mathrm{SPO}_{2}$ will not be simultaneous but will proceed in accordance with the stepped-wedge design (Fig. 1). All health facilities will have a run-in period during which nurses will be trained in pulse oximetry, $\mathrm{O}_{2}$ management, and the study protocol. Data collection (prospective enrolment and accurate electronic record-keeping) will be in place by the end of the run-in period. $\mathrm{SPO}_{2}$ will be introduced one site at a time, on a monthly basis, until all 20 sites have $\mathrm{SPO}_{2}$ installed. The timing of installation of $\mathrm{SPO}_{2}$ will be random, with allocation concealment. Analysis will compare mortality before and after implementation of $\mathrm{SPO}_{2}$, using linear mixed effects (LME) logistic regression, adjusting for the confounding effects of time (fixed effect) and site (random effect) [17]. Data collection will continue until the trial's termination, considered to be one month after the installation of $\mathrm{SPO}_{2}$ in the 20th site. 


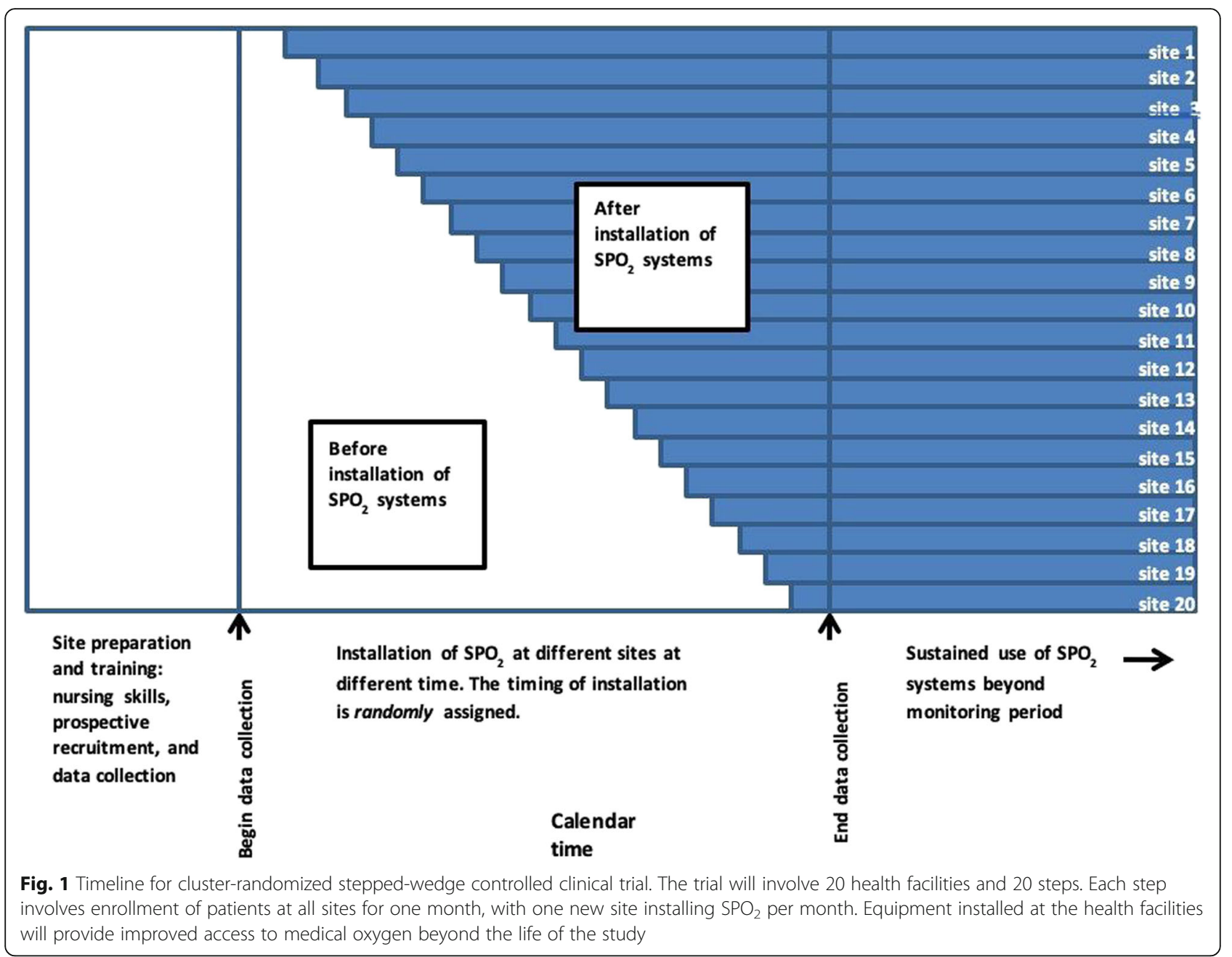

\section{Setting}

Uganda counts approximately 145,000 child deaths annually, of which $16 \%$ are attributed to pneumonia [4]. Uganda's public healthcare system consists of health centers (levels II-IV), general/provincial hospitals, regional referral hospitals, and the National Referral Hospital. The majority of deaths occur in a limited number of general hospitals and level IV health centers where at least half have poor access to a stable electrical supply or O2 cylinders [18]. These represent ideal sites for early implementation of $\mathrm{SPO}_{2}$ because of a context-specific need for an $\mathrm{O}_{2}$ delivery system that does not rely on electrical power or cylinder distribution. We will enroll 20 sites in our cluster-randomized trial.

\section{Site selection}

Health facilities were evaluated and selected based on the following criteria. Sites were included if they: (1) had pediatric inpatient services; (2) lacked consistent $\mathrm{O}_{2}$ supply on pediatric wards; and (3) had adequate space and willingness to install solar panels, a battery bank, and $\mathrm{O}_{2}$ concentrator on the hospital premises (i.e. on pediatric ward). Sites were excluded from the study if: (1) had no inpatient services for children; (2) had pre-existing, functional, and consistent $\mathrm{O}_{2}$ delivery systems (cylinder or concentrator); (3) did not have space or were unwilling to install solar panels, a battery bank, and $\mathrm{O}_{2}$ concentrator.

We conducted site visits to 44 health facilities across the country and interviewed key informants at each site. Based on site selection criteria, five sites were chosen from each of Uganda's four geographic regions, for a total of 20 sites (Fig. 2). Additional "back-up" sites have also been identified, in case of difficulties with site reengagement, site/personnel recruitment, or installation at one or more of the primary sites. Characteristics of the 20 chosen sites were as follows. While cylinder oxygen was available at 9/20 (45\%) of sites, dedicated cylinders were not available on the pediatric wards of any health facility. Oxygen concentrators were present at 18/ 20 (90\%) of sites, with only $4 / 18(22 \%)$ of sites with dedicated concentrators available on pediatric wards. Concentrators were shared across wards in 11/18 (61\%) 

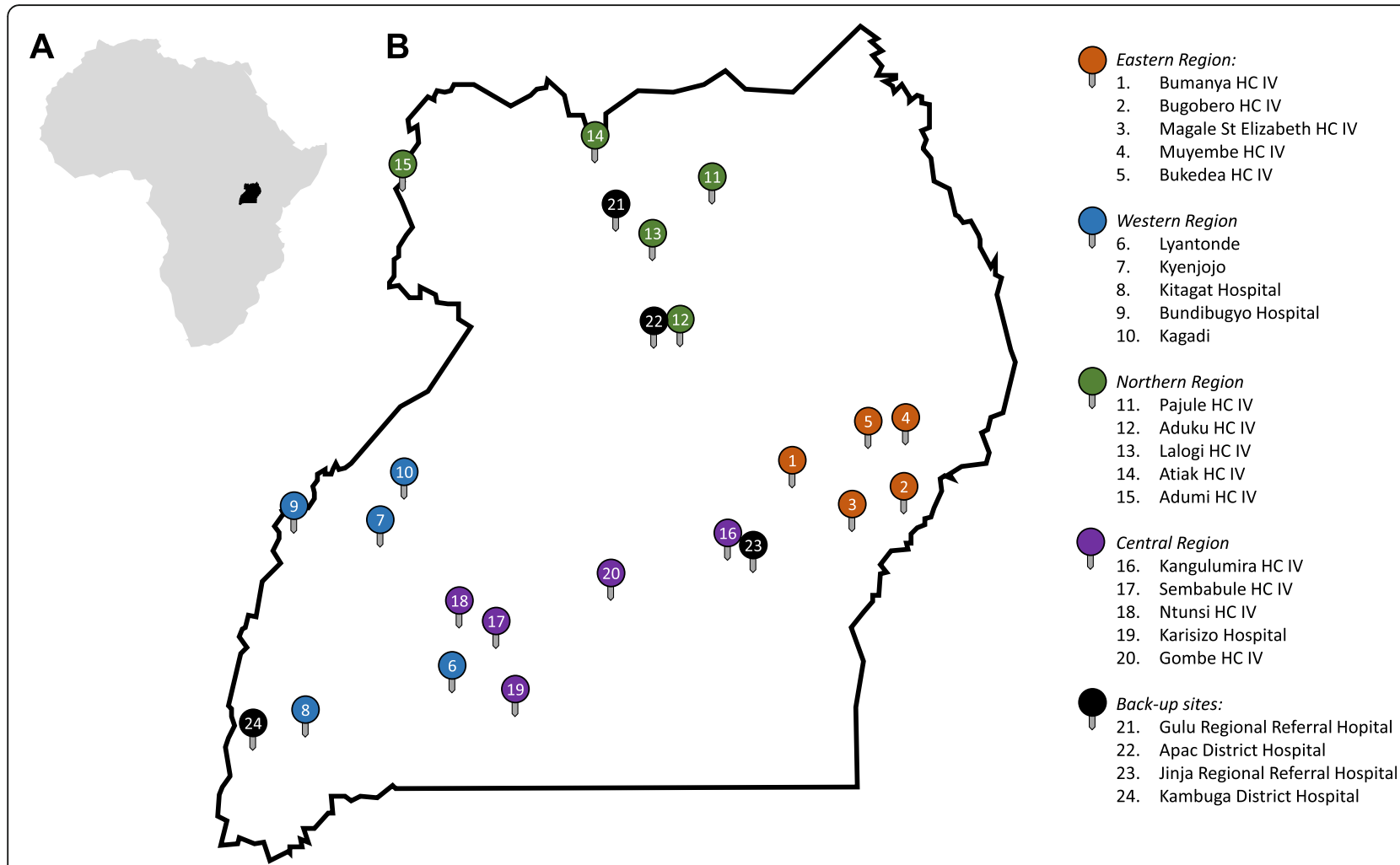

Fig. 2 Sites for implementation and evaluation of solar powered $\mathrm{O}_{2}\left(\mathrm{SPO}_{2}\right)$ delivery. a Map of Africa showing Uganda (black). b Map of Uganda showing $\mathrm{SPO}_{2}$ sites. Between four and six health facilities (District Hospitals or Level 4 Health Centers, HC IV) were chosen from each region (Central, Western, Eastern, and Northern) using the following criteria: facility has a pediatric inpatient ward; consistent $\mathrm{O}_{2}$ supply on pediatric ward is lacking; adequate space and willingness to install $\mathrm{SPO}_{2}$ system on pediatric ward. Sites are geographically distributed across the country and are likely representative of health facilities in sub-Saharan Africa where $\mathrm{SPO}_{2}$ would be cost-effective

sites. At the time of the interview, $11 / 18(61 \%)$ concentrators were operational, and only $4 / 18(22 \%)$ produced oxygen with $\geq 80 \%$ purity. Power outages were reported as frequent in $17 / 20$ (85\%) of sites. While 19/20 (95\%) of sites had generators available, none turned them on when children needed oxygen. Because electricity is necessary to run oxygen concentrators, this suggested that access to a reliable power source is a major obstacle in delivering oxygen therapy. Overall, only 3/20 (15\%) of sites had oxygen available for pediatric use.

Qualified site champions (local nursing leads) were recruited for each site to support $\mathrm{SPO}_{2}$ implementation.

\section{Participants}

We will include patients presenting to the selected sites meeting the following inclusion criteria: (1) age < 5 years; (2) hypoxemia $\left(\mathrm{SPO}_{2}<92 \%\right)$ based on non-invasive pulse oximetry taken within $24 \mathrm{~h}$ of admission, on room air; and (3) warrant hospital admission based on clinical judgement. Patients will be excluded from the study if: (1) measured $\mathrm{SPO}_{2} \geq 92 \%$; (2) they can be managed as an outpatient; (3) parents or guardians unwilling to provide written informed consent to participate in study; (4) known cyanotic cardiac condition; and (5) diagnosis of hypoxic ischemic encephalopathy in a neonate.

Screening procedures will be as follows: children aged $<5$ years presenting to the participating facilities will be screened for hypoxemia using a portable pulse oximeter if they have cough and/or difficulty breathing. Past medical history will be reviewed with the parent or guardian for known cyanotic cardiac condition and/or hypoxic ischemic encephalopathy. If the patient meets eligibility criteria, the parent or guardian will be approached for informed consent.

\section{Randomization method}

The random order of site installation will be generated before the trial. Site names will be written on paper and placed in sequentially numbered sealed opaque envelopes. Once a month, at the time of site selection for the next $\mathrm{SPO}_{2}$ installation, the next envelope will be opened to reveal the site. The envelope will be signed and dated, and all envelopes and records will be kept for quality monitoring purposes.

Randomization will be in blocks of four. Each block will include one site from each of four geographic 
regions of the country (Northern, Eastern, Western, and Central Regions). The order of the four regions within a block will be random; the selection of a site within the region will be randomly sampled without replacement. Thus, after each sequential block of four, the sites will be balanced by region.

\section{Study procedures}

For eligible participants, the parent or legal guardian will be approached for consent to participate in the study (Additional file 3). If granted, the patient will be admitted to the pediatric ward, where there will be a $\mathrm{SPO}_{2}$ system installed or not, according to random timing of installation at each site. All patients will receive standard care for their underlying disease, including antibiotics for pneumonia, intravenous fluids as necessary, blood transfusion as necessary, antipyretics, and any other medical therapy required. The study will ensure there are no stock-outs of essential medications or equipment. Basic demographic and clinical data will be collected from the case admission record, and patients will be followed during their hospital admission. The primary outcome, death at $48 \mathrm{~h}$ after admission, will be recorded. Other outcomes could include death after $48 \mathrm{~h}$, discharge, and transfer to another facility. Death at $48 \mathrm{~h}$ (versus other) will later be analyzed as a binary variable. Patients discharged or transferred to another facility will be followed up by telephone at $48 \mathrm{~h}$ after admission to assess status.

During hospitalization, secondary outcomes will be recorded, including vital signs at admission and daily thereafter until discharge. Oxygen saturations will be monitored on a 4-hourly basis, during oxygen administration and after. Oxygen utilization (flow rate, duration) will be recorded throughout hospitalization. The need for oxygen therapy will be assessed daily, using standardized criteria for weaning oxygen. The length of stay among survivors will be recorded, as well as the final patient disposition (discharged without disability, discharged with disability, transferred to another facility, or death). The participant flow is illustrated in Fig. 3.

\begin{tabular}{|c|c|c|c|c|c|c|}
\hline \multirow[b]{2}{*}{ TIMEPOINT } & \multirow{2}{*}{$\begin{array}{c}\text { Enrolment } \\
0 \\
\text { admission }\end{array}$} & \multirow{2}{*}{$\begin{array}{c}\text { Allocation } \\
0 \\
\text { admission }\end{array}$} & \multicolumn{3}{|c|}{ Post-allocation } & \multirow{2}{*}{$\begin{array}{c}\begin{array}{c}\text { Close- } \\
\text { out }\end{array} \\
t_{x}\end{array}$} \\
\hline & & & 0 & $48 \mathrm{~h}$ & discharge & \\
\hline \multicolumn{7}{|l|}{ ENROLMENT: } \\
\hline Eligibility screen & $x$ & & & & & \\
\hline Informed consent & $x$ & & & & & \\
\hline Allocation & & $x$ & & & & \\
\hline \multicolumn{7}{|l|}{ INTERVENTIONS: } \\
\hline $\mathrm{SPO}_{2}$ & & & $\leftarrow$ & & $\longrightarrow$ & \\
\hline Standard care & & & 2 & & $\longrightarrow$ & \\
\hline \multicolumn{7}{|l|}{ ASSESSMENTS: } \\
\hline $\begin{array}{l}\text { Demographics, } \\
\text { History of } \\
\text { presenting illness, } \\
\text { Past medical } \\
\text { history, Physical } \\
\text { exam findings }\end{array}$ & & $\mathrm{X}$ & & & & \\
\hline Mortality (48h) & & & & $x$ & & \\
\hline $\begin{array}{l}\text { Length of stay, } \\
\text { Duration of } \mathrm{O}_{2} \\
\text { therapy }\end{array}$ & & & & & $\mathrm{x}$ & \\
\hline $\begin{array}{l}\mathrm{O}_{2} \text { system failures, } \\
\text { Costs }\end{array}$ & & & & & & $\mathrm{x}$ \\
\hline
\end{tabular}

Fig. 3 Participant schedule in accordance with SPIRIT 2013 guidelines. The flow diagram identifies the time points during the study including Enrolment (admission), Allocation (admission), post-allocation (0, $48 \mathrm{~h}$, discharge), and close-out (end of study). Relevant actions of enrolment, interventions, and assessments performed at the respective time points are marked with an $\mathrm{X}$ on the diagram 


\section{Outcome measures}

Our study's primary endpoint is mortality at $48 \mathrm{~h}$ after admission. We will also evaluate several secondary outcome measures, including in-hospital mortality (time to death), length of hospital stay, duration of supplemental $\mathrm{O}_{2}$ therapy (time to wean $\mathrm{O}_{2}$ ), $\mathrm{O}_{2}$ delivery system failure(s), cost (installation, servicing, and maintenance), baseline and retained nursing skills in managing oxygen therapy, etiology (based on polymerase chain reaction of dried blood sample and nasopharyngeal swabs), as well as diagnostic and prognostic host biomarkers (Additional file 4).

\section{Data monitoring}

Data will be routinely monitored on each site by a dedicated data collection officer or study nurse, who will be responsible for recording patient information, follow-up, and uploading data to trial databases through KoBoCollect on tablet PCs. During entry, any missing data will be noted on an Errors/Omission log which will be checked regularly by the study team to fill in the missing data. Once uploaded, study data will be securely stored in a locked room and on a password-protected device, accessible only to study personnel. The study monitor or other authorized representatives may inspect all documents and records required to be maintained by the principal investigator; the study site will permit access to such records.

Adverse events (AEs) and unintended effects of $\mathrm{SPO}_{2}$ will be monitored, reported, and collected by clinical staff, and will be managed according to national and local standard of care. AEs will be monitored continuously during the study by a dedicated nursing staff. A running $\log$ of AEs will be kept and reviewed periodically to allow the study team to assess if any patterns of AEs emerge in real time. All study deaths will require completion of a serious $\mathrm{AE}$ form, which will be made available to ethics boards and regulatory authorities within seven days of the event.

No interim data analysis or trial stopping rules are planned for this trial. Oxygen is known to be an essential and life-saving therapy and the efficacy of the $\mathrm{SPO}_{2}$ system has been shown to be non-inferior compared to conventional oxygen therapy in the Ugandan context [15]. The stepped-wedge design and commitment to participating sites demands that $\mathrm{SPO} 2$ be installed at all sites, such that trial discontinuation at midpoint is not feasible. Because we do not plan to halt the trial before enrolling the planned number of clusters, we have not planned for a Data Monitoring Committee (DMC).

\section{Statistical considerations}

In our trial, we have a cluster-randomized design. The primary dependent variable is the 48 -h mortality. The independent (predictor) variable of interest is the exposed/ unexposed status of an individual patient (fixed effect), which will depend on whether the patient presents to a participating site before or after installation of $\mathrm{SPO}_{2}$. Furthermore, we have covariates which will be modeled as both fixed and random effects. We will use a LME logistic regression model to examine the effect of $\mathrm{SPO}_{2}$ on mortality while adjusting for changes in mortality over time (fixed effect) and variability in mortality between sites (random effect). We will use $R$ [19] and lme4 [20] to model the binary outcome (survival) as a function of $\mathrm{SPO}_{2}$ treatment, calendar time, and site. As fixed effects, we will enter $\mathrm{SPO}_{2}$ treatment (before or after $\mathrm{SPO}_{2}$ installation) and calendar time, without interaction term, into the model. We will model site as a random effect. We will determine the statistical significance of the $\mathrm{SPO}_{2}$ treatment effect comparing models with and without the $\mathrm{SPO}_{2}$ treatment term. If the model fit is significantly improved with inclusion of the treatment term, at $\alpha=0.05$ level of significance, we will conclude that the $\mathrm{SPO}_{2}$ treatment effect is statistically significant. We will estimate the $95 \%$ confidence interval of the treatment effect (odds ratio) from the coefficient of the LME logistic regression model.

Secondary outcomes will be assessed using descriptive and comparative statistics, as appropriate. Where possible, LME models accounting for the clustered data structure and effects of calendar time will be used to determine differences between $\mathrm{SPO}_{2}$-treated and untreated patients, with necessary adjustments being made to allow for multiple comparisons within secondary outcomes.

\section{Sample size}

We will include 20 health facilities and a total of 2400 hospitalized patients. This sample size was calculated using a computer simulation, modeling trial conditions and applying the planned statistical analysis. Although analytic methods for sample size calculation for clusterrandomized stepped-wedge trials have been published [21], these require estimates of nuisance parameters (e.g. intra-cluster correlation coefficient) which were not available in the Ugandan context, such that the validity of this method was unknown.

For simulation parameters, we used data from the Demographic and Health Information System (DHIS) 2015 for Uganda. This database contains information on the number of patients with clinical pneumonia admitted to each hospital and the number of fatal cases, allowing us to estimate site-specific pneumonia mortality. In a computer simulation, we randomly selected 20 representative sites from the DHIS and used the number of patients admitted with pneumonia at each site to estimate the incidence of hypoxemic patients (13\% [22]) that would be enrolled in a hypothetical clinical trial. The monthly number of hypoxemic pneumonia admissions was modeled as a Poisson distribution [23]. Monthly random allocation of 
$\mathrm{SPO}_{2}$ to the 20 sites was simulated, according to the stepped-wedge design. The outcome of each patient at $48 \mathrm{~h}$ was modeled as a Bernoulli trial with probability of fatal outcome equal to the site-specific mortality. We used DHIS 2015 data to estimate the baseline mortality assuming a mortality reduction of $35 \%$ after installation of $\mathrm{SPO}_{2}$ [13]. Having generated simulated trial data, we ran the planned statistical analysis, fitting a LME logistic regression model, as described above. Mortality was modeled as a function of $\mathrm{SPO}_{2}$ treatment, with adjustment for covariates of calendar time (fixed effect) and site (random effect), and the $p$ value for the treatment effect was calculated. We repeated the simulation $>5000$ times and determined the proportion of trials that correctly detected a difference between patients receiving $\mathrm{SPO}_{2}$ and those not receiving $\mathrm{SPO}_{2}$ (statistical power). Using this simulation strategy, 20 sites enrolling a total of 2400 patients would provide $>80 \%$ power to detect a $35 \%$ mortality benefit of $\mathrm{SPO}_{2}$ at the $\alpha=0.05$ level of significance [17, 24]. Data collection could feasibly be completed within 24 months (Fig. 4).

Figure 4a shows the statistical power as a function of the number of sites and the duration of enrolment. Each power estimate was based on at least 100 hypothetical trials in a computer simulation. Approximately 20 sites were required, enrolling patients for two years, to achieve sufficient statistical power. Figure $4 \mathrm{~b}$ shows individual trial simulations, illustrating increasing power with increasing sample size. Figure 4c shows the sensitivity of the study power to input parameters: baseline mortality and mortality reduction with $\mathrm{SPO}_{2}$.

\section{Discussion}

The global burden of pneumonia mortality is concentrated in resource-constrained settings in Africa and Asia [2, 25-27]. Access to $\mathrm{O}_{2}$ remains limited and the need for $\mathrm{O}_{2}$ has come to the forefront of global health priorities with the WHO recently adding $\mathrm{O}_{2}$ to its Model List of Essential Medicines [28]. Several countries, such as Nigeria and Ethiopia, have begun to develop national frameworks for $\mathrm{O}_{2}$ scale-up [29, 30]. With increasing recognition of the importance of $\mathrm{O}_{2}$ therapy in lowresource settings, novel methods of $\mathrm{O}_{2}$ delivery are required. $\mathrm{SPO}_{2}$ offers a reliable and sustainable source of $\mathrm{O}_{2}$ that could have utility in these low-resource settings. We hypothesize that we will observe a reduction in mortality in children hospitalized with hypoxemia, after installation of $\mathrm{SPO}_{2}$, relative to current level of care. We will test this hypothesis in a stepped-wedge clusterrandomized study in Uganda.

$\mathrm{SPO}_{2}$ has a number of benefits that lend themselves to further scale-up: reliability in settings with poor access

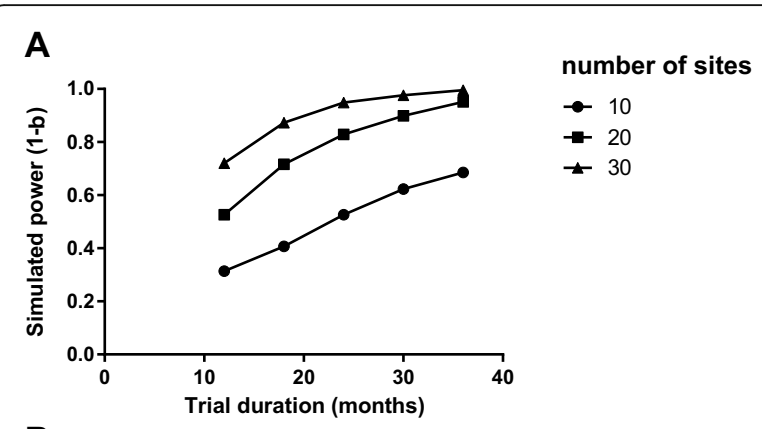

B

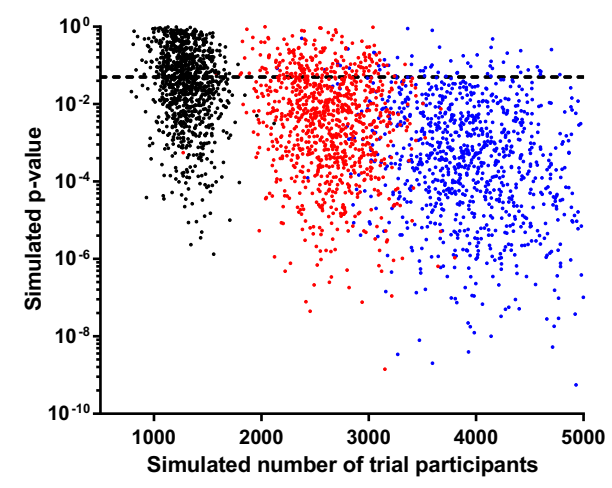

C

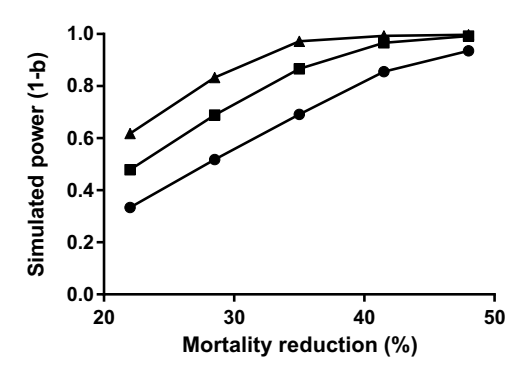

baseline mortality

$\rightarrow 10 \%$

$\rightarrow-15 \%$

$\rightarrow 20 \%$

Fig. 4 Computer simulation for sample size estimation. a In the simulation, study power varied with the number of clusters and the duration of enrolment, as expected. Approximately 20 sites enrolling patients over 24 months would provide power of $80 \%$. Each dot represents at least 100 simulated trials. b Each dot represents one simulated trial with 20 sites and 20 steps, enrolling patients for two weeks (black dots), four weeks (red dots), or six weeks (blue dots) at each step. The study power is approximated by the proportion of trials appropriately detecting a statistically significant effect of $\mathrm{SPO}_{2}$ ( $p<0.05$, dotted line). The simulation was repeated 3000 times to generate a plot of $p$ value and number of trial participants. For trial simulations with four-week steps (total duration 21 months), the median number of participants enrolled was 2600 (interquartile range $2400-2900$ ) and the statistical power was $82 \%$. c In the simulation, study power was also sensitive to variations in the assumptions of baseline mortality and mortality reduction. Our base case (15\% baseline mortality, mortality reduction of $35 \%$ ) was associated with statistical power of $80 \%$. Each dot represents at least 100 simulated trials

to electricity or $\mathrm{O}_{2}$ cylinders [31]; abundance of solar radiation in target LMICs in the tropics [32]; minimal maintenance and training requirements [32]; and cost- 
effectiveness after a one-time capital investment for installation of equipment. Our group has already demonstrated the feasibility and effectiveness of $\mathrm{SPO}_{2}$ at two resource-limited hospitals $[14,15]$. The proposed study will demonstrate mortality benefit of $\mathrm{SPO}_{2}$ in a countrywide roll-out, providing key data for decision-makers within public health systems with respect to utility (lives saved) and costs of this technology. To our knowledge, only one previous study has quantified the effect size of improved $\mathrm{O}_{2}$ delivery on child mortality in a low-resource context [13]. Additional findings from this trial will be of broad interest, including: pneumonia-related mortality at various thresholds of hypoxemia [11]; oxygen utilization at representative health facilities; engineering aspects and costs (installation and maintenance) of $\mathrm{SPO}_{2}$ implemented across an entire country; and training strategies in oxygen delivery for frontline nurses.

The choice of study design is motivated by ethical and pragmatic considerations [24,33]. The cluster-randomized trial design is modern, innovative, and particularly suited to investigations of community level public health interventions that have been proven effective in individual level trials (i.e. "phase IV" effectiveness trials) [17, 24]. Staged roll-out of $\mathrm{O}_{2}$ delivery avoids the ethical concern of randomizing individuals to a "no $\mathrm{O}_{2}$ " arm. All facilities will transition from control to intervention groups [24, 34], providing permanent access to previously unavailable $\mathrm{O}_{2}$ therapy by the end of the trial. The staged approach will ease logistical constraints otherwise associated with implementing interventions all together at the same time [24]. With respect to scientific validity, our statistical plan (LME logistic regression model) will adjust for variability in mortality between different sites (random effect) and temporal trends in mortality (fixed effect). Some risks associated with this design were highlighted in a recent systematic review of 46 individual stepped-wedge studies: clusters dropped after randomization or after data collection had started (six studies); delay in implementation of interventions (four studies); and clusters not receiving the intervention at all (five studies) [33]. To mitigate this risk, we have selected two back-up sites that may be recruited in case of cluster dropout. Although this mitigating strategy compromises the stepped-wedge design, it may be necessary to achieve the desired sample size. In case of cluster dropout and addition of new clusters, the statistical plan will include sensitivity analyses including sites planned $a$ priori and a pragmatic analysis of all sites ultimately included.

Our study sample size was calculated via a computer simulation using real-world parameters using the Uganda DHIS [22, 35], data from previous large-scale studies [31], and systematic reviews [22]. We applied our planned primary analysis to the simulated data for > 5000 trials to estimate a required sample size of 2400 patients from 20 sites, with 20 steps, recruited over two years to detect a mortality benefit estimated at 35\% with $80 \%$ power $(\alpha=0.05)$. Other published stepped-wedge trials in a recent systematic review were of comparable size: median number of participants of 1720 (range 16292,000), 2-190 clusters, 2-15 steps, over a median period of 20 months (range 9 days to 4 years). Our approach, using computer simulation, has been recommended by previous authors, who noted advantages of simulation-based methods to overcome some of the limitations of analytical formulae and in dealing with the specific features of the study [36].

In addition to our scientific objectives, this study aims to build capacity among frontline nurses for monitoring and delivery of supplemental $\mathrm{O}_{2}$ therapy, including pulse oximetry. A study conducted to assess pulse oximetry knowledge and training needs in ward nurses and doctors concluded that only $16 \%$ of participants had received any formal training for pulse oximetry and $65 \%$ of the participants expressed the need for more training [37]. In another report from Uganda, most nurses were comfortable with the use of oxygen concentrators but were not familiar with pulse oximetry [6]. To address this gap, recognizing context-specific challenges of implementing pulse oximetry [38], $\mathrm{SPO}_{2}$ study will train nursing staff in pulse oximetry and safe delivery of $\mathrm{O}_{2}$. With enhanced knowledge, skills, and availability of equipment (pulse oximeters and $\mathrm{SPO}_{2}$ equipment), our study will improve the quality of care provided to hypoxemic patients during the study and after the study has ended.

Our study has several limitations. The multi-center stepped-wedge design involving health facilities within the public sector, is vulnerable to some external challenges that may not be directly influenced by the researchers (e.g. change of hospital staff or withdrawal of clusters from the study) [33, 39]. The health facilities participating in this study will be in rural or remote communities in Uganda, such that generalizability of our findings to urban or resource-intensive facilities will be limited. The etiology of hypoxemic illness will not be precisely identified. Radiographic confirmation of pneumonia and microbiological confirmation of the infectious etiology would be desirable, but the requisite radiology and lab services are not available in most centers where $\mathrm{SPO}_{2}$ will be installed. Additional laboratory testing such as blood culture, sputum culture, viral studies, and a panel of infectious diseases serology would be necessary to definitively diagnose conditions such as pneumococcal pneumonia and viral respiratory tract infections. However, resource limitations preclude an exhaustive battery of diagnostic tests in our setting. Although precise microbiological etiology may not be known, mortality (primary outcome) as well as length of stay (secondary outcome) are clinically meaningful outcomes that can be assessed unambiguously in the 
context of our study. This study will allow us to demonstrate the mortality benefit of $\mathrm{SPO}_{2}$ for treatment of hypoxic respiratory illness, irrespective of etiology, and is similar to the diagnostic capacity of other health facilities where $\mathrm{SPO}_{2}$ would potentially be implemented.

Given the magnitude of pediatric pneumonia deaths, estimated at 900,000 per year [40], a life-saving, costeffective intervention such as $\mathrm{SPO}_{2}$ could represent an important tool for the improvement of global child survival. Demonstrating a mortality benefit of $\mathrm{SPO}_{2}$ will provide strong supportive evidence for the system and could catalyze the widespread implementation of $\mathrm{SPO}_{2}$ in resource-limited settings across Africa and Asia.

\section{Trial status}

Currently recruiting.

Protocol version number 2.2, dated 21 April 2019

Date that recruitment began: 1 July 2019

Anticipated date that recruitment will be completed: 1 July 2021

\section{Supplementary information}

Supplementary information accompanies this paper at https://doi.org/10. 1186/s13063-019-3752-2.

Additional file 1. World Health Organization Trial Registration Data Set.

Additional file 2. Spirit Checklist.

Additional file 3. Consent Form.

Additional file 4. Biological Specimens \& Ancillary Studies.

\section{Abbreviations}

DHIS: Demographics and Health Information Systems; LME: Linear mixed effects; LMICs: Low- and middle-income countries; $\mathrm{O}_{2}$ : Oxygen; $\mathrm{SPO}_{2}$ : Solarpowered oxygen

\section{Acknowledgements}

We thank the participants of our past studies of $\mathrm{SPO}_{2}$, which provided the evidence basis for this country-wide study.

\section{Authors' contributions}

NC and MTH wrote the first draft of the manuscript. QM, ALC, SN, LH, and $\mathrm{ROO}$ contributed to the study design and critically reviewed the manuscript. MTH designed the study, obtained the funding, performed the computer simulation for the sample size calculation, and critically reviewed the manuscript. All authors read and approved the final manuscript.

\section{Funding}

Grand Challenges Canada (Grant Number 1909-27795) and The Women and Children's Health Research Institute (Reference Number WCHSSLDRP 2371) provided funding for the trial. The funders had no role in writing the protocol or the current manuscript. The funders will have no role in the design of the study and collection, analysis, interpretation of data, or writing the report of study findings and subsequent publications.

\section{Availability of data and materials}

De-identified individual clinical trial participant level data will be shared upon reasonable request submitted to the corresponding author. Findings will be disseminated through presentations at international conferences and will be published in open-access peer-reviewed journals for broad readership and to ensure that the data are available to health workers in low-and middle-income countries. At the conclusion of the study, we will hold a "town hall" meeting at participating health facilities, where local and global study data will be shared with the community members, with opportunities to ask questions.

Code ( $\mathrm{R}$ program) for the computer simulation is available from the corresponding author on reasonable request.

\section{Ethics approval and consent to participate}

The School of Biomedical Sciences Research Ethics Committee, Makerere University, Uganda, (reference SBS 644; Approved: 5 March 2019) and the Health Sciences Research Ethics Committee, University of Alberta, Canada (reference Pro00084784; Approved: 25 February 2019) have approved the study. Amendments and modifications to the trial protocol will be forwarded to the aforementioned organizations for review and approval within two weeks of the changes. Written informed consent will be obtained from the parent or guardian of all participants (Additional file 3).

\section{Consent for publication}

Not applicable.

\section{Competing interests}

MTH, ROO, SN, QM, and ALC are listed as inventors on New International (PCT) Patent Application Serial No. PCT/CA2018/051151 and United States Provisional Patent Application Serial No.62/559,702

\section{Author details}

${ }^{1}$ Department of Pediatrics, University of Alberta, 3-588D Edmonton Clinic Health Academy, 1140587 Ave NW, Edmonton, Alberta T6G 1C9, Canada. ${ }^{2}$ Kabale Regional Referral Hospital, Kabale, Uganda. ${ }^{3}$ Indiana University, Indianapolis, USA. ${ }^{4}$ Kelowna General Hospital, Kelowna, Canada. ${ }^{5}$ Ministry of Health, Kampala, Uganda. 'Department of Paediatrics and Child Health, Mulago Hospital and Makerere University, Kampala, Uganda. ${ }^{7}$ Department of Medical Microbiology and Immunology, University of Alberta, Edmonton, Canada. ${ }^{8}$ School of Public Health, University of Alberta, Edmonton, Canada. ${ }^{9}$ Stollery Science Lab, Edmonton, Canada. ${ }^{10}$ Women and Children's Health Research Institute, Edmonton, Canada.

Received: 27 July 2019 Accepted: 24 September 2019

Published online: 05 December 2019

\section{References}

1. Bawaskar HS. The world's forgotten children. Lancet. 2003;361(9364):1224-5.

2. Black RE, Morris SS, Bryce J. Where and why are 10 million children dying every year? Lancet. 2003;361(9376):2226-34.

3. Nascimento-Carvalho CM. Etiology of childhood community acquired pneumonia and its implications for vaccination. Braz J Infect Dis. 2001;5(2): $87-97$.

4. UNICEF. Situational Analysis of Children in Uganda. Geneva: UNICEF; 2015 p. 1-179. Available at:https://www.unicef.org/uganda/media/1791/file/ Situation\%20analysis\%20of\%20children\%20in\%20Uganda\%20.pdf. Accessed 18 Sept 2019.

5. Coghlan B, Brennan RJ, Ngoy P, Dofara D, Otto B, Clements M, et al. Mortality in the Democratic Republic of Congo: a nationwide survey. Lancet. 2006:367(9504):44-51.

6. Nabwire J, Namasopo S, Hawkes M. Oxygen availability and nursing capacity for oxygen therapy in Ugandan paediatric wards. J Trop Pediatr. 2018:64(2):97-103.

7. Belle J, Cohen H, Shindo N, Lim M, Velazquez-Berumen A, Ndihokubwayo $\mathrm{JB}$, et al. Influenza preparedness in low-resource settings: a look at oxygen delivery in 12 African countries. J Infect Dev Ctries. 2010;4(7):419-24.

8. Bradley B, Light J, Ebonyi A, N'Jai P, Idea R, Ebruke B, et al. Implementation and 8-year follow-up of an uninterrupted oxygen supply system in a hospital in The Gambia. Int J Tuberc Lung Dis. 2016;20(8):1130-4.

9. Hill SE, Njie O, Sanneh M, Jallow M, Peel D, Njie M, et al. Oxygen for treatment of severe pneumonia in The Gambia, West Africa: a situational analysis. Int J Tuberc Lung Dis. 2009;13(5):587-93.

10. Howie SR, Hill S, Ebonyi A, Krishnan G, Njie O, Sanneh M, et al. Meeting oxygen needs in Africa: an options analysis from the Gambia. Bull World Health Organ. 2009;87(10):763-71

11. Maitland K, Kiguli S, Opoka RO, Olupot-Olupot P, Engoru C, Njuguna P, et al. Children's Oxygen Administration Strategies Trial (COAST): a randomised controlled trial of high flow versus oxygen versus control in African children with severe pneumonia. Wellcome Open Res. 2017;2:100. 
12. Adair-Rohani H, Zukor K, Bonjour S, Wilburn S, Kuesel AC, Hebert R, et al Limited electricity access in health facilities of sub-Saharan Africa: a systematic review of data on electricity access, sources, and reliability. Glob Health Sci Pract. 2013;1(2):249-61.

13. Duke T, Wandi F, Jonathan M, Matai S, Kaupa M, Saavu M, et al. Improved oxygen systems for childhood pneumonia: a multihospital effectiveness study in Papua New Guinea. Lancet. 2008;372(9646):1328-33.

14. Turnbull H, Conroy A, Opoka R, Namasopo S, Kain K, Hawkes M. Solarpowered oxygen delivery: proof of concept. Int J Tuberc Lung Dis. 2016; 20(5):696-703.

15. Hawkes MT, Conroy AL, Namasopo S, Bhargava R, Kain KC, Mian Q, et al. Solar-powered oxygen delivery in low-resource settings: a randomized clinical noninferiority trial. JAMA Pediatr. 2018;172(7):694-6.

16. Hawkes MT, Opoka RO. Solar Powered Oxygen Delivery: an open label noninferiority randomized comparison to standard oxygen delivery. 2015. Available at: https://linicaltrials.gov/ct2/show/NCT02100865. Accessed 18 Sept 2019

17. Hussey MA, Hughes JP. Design and analysis of stepped wedge cluster randomized trials. Contemp Clin Trials. 2007;28(2):182-91.

18. Ministry of Health of Uganda. Demographic and health information system. Kampala: $\mathrm{MoH} ; 2015$

19. R: A language and environment for statistical computing. https://www.Rproject.org/. Accessed 18 Sept 2019.

20. Bates $D$, Mächler $M$, Bolker BM, Walker SC. Fitting linear mixed-effects models using Ime4. J Stat Softw. 2015;67(1):1-48.

21. Hooper R, Teerenstra S, de Hoop E, Eldridge S. Sample size calculation for stepped wedge and other longitudinal cluster randomised trials. Stat Med. 2016:35(26):4718-28

22. Subhi R, Adamson M, Campbell H, Weber M, Smith K, Duke T, Group HiDCS. The prevalence of hypoxaemia among ill children in developing countries: a systematic review. Lancet Infect Dis. 2009;9(4):219-27.

23. Barra M, Lindstrom JC, Adams SS, Augestad LA. Seasonally adjusted birth frequencies follow the poisson distribution. Tidsskr Nor Laegeforen. 2015; 135(23-24):2154-8

24. Hemming K, Haines TP, Chilton PJ, Girling AJ, Lilford RJ. The stepped wedge cluster randomised trial: rationale, design, analysis, and reporting. BMJ. 2015; 350:h391.

25. Kaplan W, Wirtz VJ, Mantel-Teeuwisse A, Stolk P, Duthey B, Laing R. Priority medicines for Europe and the world 2013 update. Geneva: WHO; 2013.

26. McAllister DA, Liu L, Shi T, et al. Global, regional, and national estimates of pneumonia morbidity and mortality in children younger than 5 years between 2000 and 2015: a systematic analysis. The Lancet Global Health. 2019;7(1):e47-57.

27. Wardlaw T, You D, Newby H, Anthony D, Chopra M. Child survival: a message of hope but a call for renewed commitment in UNICEF report. Reprod Health. 2013;10:64.

28. WHO. WHO model list of essential medicines for children. 6th ed. Geneva: WHO; 2017

29. Federal Ministry of Health of Nigeria. National strategy for the scale-up of medical oxygen in health facilities 2017-2022. Abuja: Federal Ministry of Health; 2017. https://www.medbox.org/ng-drugs-med-equipment/nationalstrategy-for-the-scale-up-of-medical-oxygen-in-health-facilities-2017-2022/ preview. Accessed 18 Sept 2019

30. Ministry of Health, The Federal Democratic Republic of Ethiopia. Nationa medical oxygen and pulse oximetry scale up road map (2016-2020/21). Addis Ababa: $\mathrm{MoH}$. Available at: https://vdocuments.mx/national-medicaloxygen-and-pulse-oximetry-scale-up-road-map.html. Accessed 18 Sept 2019.

31. Duke T, Hwaihwanje I, Kaupa M, Karubi J, Panauwe D, Sa'avu M, et al. Solar powered oxygen systems in remote health centers in Papua New Guinea: a large scale implementation effectiveness trial. J Glob Health. 2017;7(1):010411.

32. Nyende S, Conroy A, Opoka RO, Namasopo S, Kain KC, Mpimbaza A, et al. Solar-powered oxygen delivery: study protocol for a randomized controlled trial. Trials. 2015;16(1):297.

33. Eichner FA, Groenwold RHH, Grobbee DE, Oude Rengerink K. Systematic review showed that stepped-wedge cluster randomized trials often did not reach their planned sample size. J Clin Epidemiol. 2019;107:89-100.

34. Spiegelman D. Evaluating Public Health Interventions: 2. Stepping Up to Routine Public Health Evaluation With the Stepped Wedge Design. Am J Public Health. 2016;106(3):453-7.

35. Arnold BF, Hogan DR, Colford JM Jr, Hubbard AE. Simulation methods to estimate design power: an overview for applied research. BMC Med Res Methodol. 2011;11:94.
36. Baio G, Copas A, Ambler G, Hargreaves J, Beard E, Omar RZ. Sample size calculation for a stepped wedge trial. Trials. 2015;16:354.

37. Davies G, Gibson AM, Swanney M, Murray D, Beckert L. Understanding of pulse oximetry among hospital staff. N Z Med J. 2003;116(1168):U297.

38. Graham HR, Bakare AA, Gray A, Ayede Al, Qazi S, McPake B, et al. Adoption of paediatric and neonatal pulse oximetry by 12 hospitals in Nigeria: a mixed-methods realist evaluation. BMJ Glob Health. 2018;3(3):e000812.

39. Kotz D, Spigt M, Arts IC, Crutzen R, Viechtbauer W. Use of the stepped wedge design cannot be recommended: a critical appraisal and comparison with the classic cluster randomized controlled trial design. J Clin Epidemiol. 2012;65(12):1249-52.

40. McAllister DA, Liu L, Shi T, Chu Y, Reed C, Burrows J, et al. Global, regional, and national estimates of pneumonia morbidity and mortality in children younger than 5 years between 2000 and 2015: a systematic analysis. Lancet. 2019;7:e47-57.

\section{Publisher's Note}

Springer Nature remains neutral with regard to jurisdictional claims in published maps and institutional affiliations.
Ready to submit your research? Choose BMC and benefit from:

- fast, convenient online submission

- thorough peer review by experienced researchers in your field

- rapid publication on acceptance

- support for research data, including large and complex data types

- gold Open Access which fosters wider collaboration and increased citations

- maximum visibility for your research: over $100 \mathrm{M}$ website views per year

At BMC, research is always in progress.

Learn more biomedcentral.com/submissions 